\title{
A portable Test Device for Magnetic Latching Relay
}

\author{
Zhongtao SHEN \\ Department of Mechanical and Electrical Engineering, Beijing Vocational College of Labour and \\ Social Security, Beijing, 100029, China \\ email:shenzht@qq.com
}

Keywords: magnetic latching relay, batch test, parameter testing, testing device

\begin{abstract}
Magnetic latching relay, with the advantages of power saving, stable performance, small cubage and large carrying capacity, has been extensively used in power quality improvement field. While the testing equipment of magnetic latching relay, with a lower work efficiency, only targets the manufacturers. To solve this problem, the article proposes a portable test device for magnetic latching relay, introduces the device frame, hardware, software implementation and the test procedure, illustrates the test project, method and result by testing cases. The testing result demonstrates that the device is technically feasible and actualizes the automatic batch testing for latching relay, and that the device runs well during the testing procedure, holds the character of convenient connection and portability and can fully satisfy the field test requirements.
\end{abstract}

\section{Introduction}

Magnetic latching relay is late-model relay which is widely used in smart meter, SVC, power quality management. It has the advantages of stable performance, small capacity, large carrying ability and cheap price. The most standing character of latching relay is that the coil is transiently excited only on the switch of working condition, while not needs to be excited during the normal working conditions. So there will consume less power [1] [2]. The reliability of latching relay keeps a close relationship with the safe function of power distribution system and electrical control system. In order to guarantee the accuracy and correctness of the product, the relay consumers who are in the fields of the power quality management need to check the parameter of the relay, which is one of the most important measures that should be taken for guaranteeing the quality and the life of the products.

The test content of magnetic latching relay includes contact resistance testing, coil resistance testing, time parameter, pull-in voltage, release voltage and coil current testing. Currently, a great deal of research has been conducted on the test principle and test method which target the function and the parameter of relay. In the meantime, different test devices have been applied in relay manufacturers with a good feasibility results [3]. However, the disadvantage of the device is that the floor area is large and the Operation procedure is complicated, and it is not convenient for the QC Assistant to check the goods when they are in stock. At present, there are few test instruments for magnetic latching relay on the market [4].

In high current and high requirement for real-time applications such as reactive power compensation and power quality management field [5], the manufacturers who using relays are most concerned about the contact resistance and time parameters. According to the test requirements mentioned above, this paper proposes a portable magnetic latching relay batch testing device, which adopts a modular design concept, through software configuration mode to realize the magnetic resistance test and time parameters test, and through the test case illustrates the test items, test methods and test results of the device.

\section{System Architecture}

The overall design of the portable test device is shown in Figure 1. 


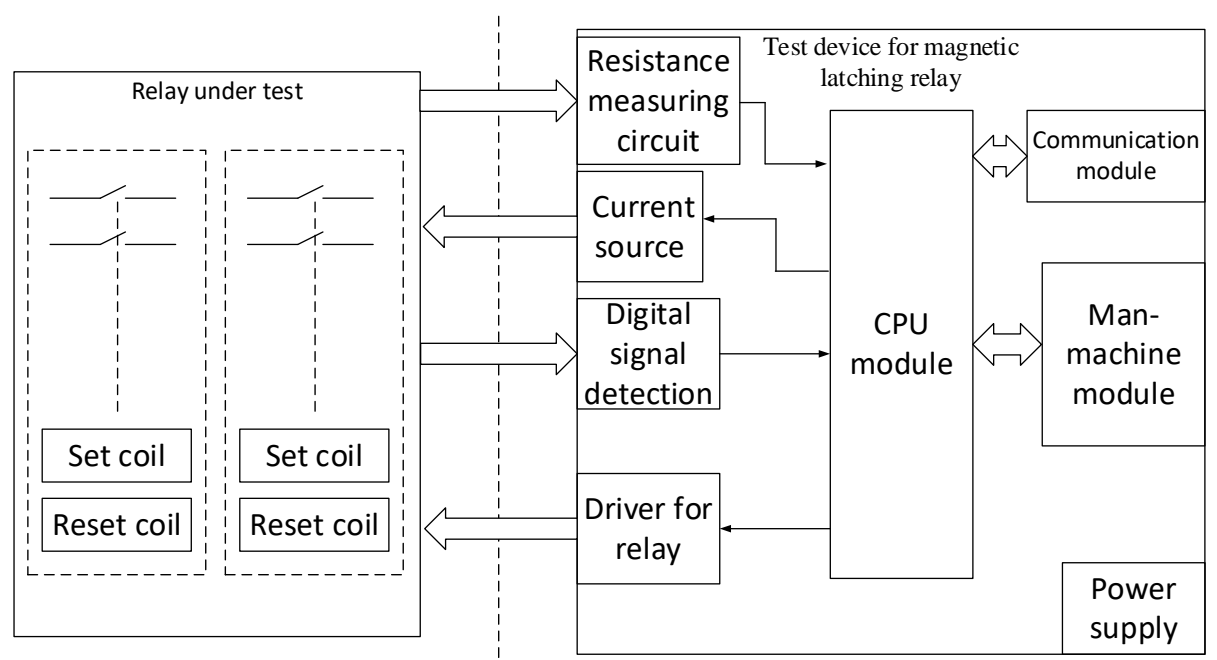

Fig.1. The overall design of the portable test device

The portable test device adopts the design method of small portable host plus portable terminal. The portable host and terminal are connected by network cable, and the batch testing of latching relay is realized. The portable case adopts the design of reinforcement, which has very strong shock resistance and has good heat dissipation performance.

The test device test time parameters and contact resistance by configuration, test results can be obtained by computer linked to the testing device, also can through the touch screen of the testing device. Test report can be automatic generated after the completion of the test system, the report not only can be output through the printer, but also can be uploaded to the cloud platform which is used for the access of vendor review.

\section{Hardware Design}

The hardware of the magnetic latching relay batch testing device mainly comprises a CPU module, a controllable constant current source, resistance measurement circuit, relay drive circuit, digital signal detection circuit, communication module, man-machine interaction module and power supply circuit.

The testing device uses the 32-bit ARM chip of ST Company as the main CPU. The chip has the interface of I/O, SPI, I2C, RS232 and Ethernet, it can communicate with touch screen and background terminal. The main function of CPU include: control of relay switching logic, control and adjustment of constant current source, calculation of relay contact resistance, measure of time parameters, communication with the man-machine module and test report generation.

According to the specifications of the relay, the controllable constant current source can generate a steady DC current of 0 30 amperes which is used to measure the resistance of relay contacts. The controllable constant current source module is mainly composed of a switching power supply, an electronic switch and a digital potentiometer. The hardware structure is shown in Figure 2. Among them: the electronic switch is used to control the on-off of the constant current source. It is achieved by a bidirectional triode thyristor which is driven by a common IO pin of CPU. The digital potentiometer is used to adjust the amplitude of the current and is driven by the CPU SPI bus.

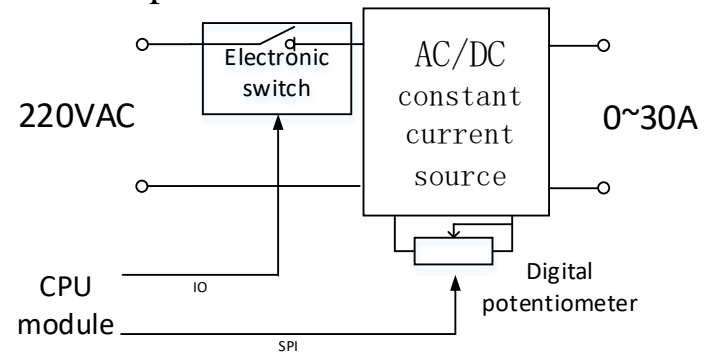

Fig.2. The overall design of the controllable constant current source 
The resistance measuring circuit calculate the resistance value of the contacts by measuring the current flowing through the contacts and the voltage across the contacts. The structure of the resistance measurement module is shown in Figure 3. The current signal and voltage signal are sent into the A/D chip after signal conditioning. The A/D chip uses a CS5532 which have internal amplifier inside to amplify current signals. The CS5532 has excellent dynamic performance and two differential inputs. It can directly measure tiny signal from the sensor and simplify the resistance measurement circuit.

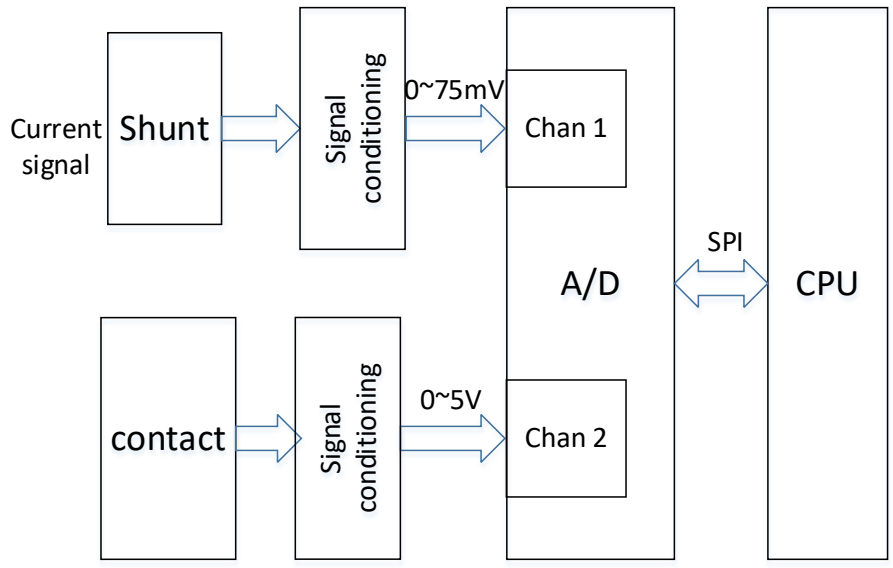

Fig.3. Schematic diagram of resistance measurement module

The function of the relay drive circuit is to receive the control instructions of the CPU and drive the coils of the relay. The circuit adopts ULN2004 as driver chip, each magnetic latching relay have a set coil and a reset coil, two coils are supplied by a power supply module.

The digital signal detection module mainly consists of optocoupler isolation circuit and shaping circuit, used to detect the contact state of relay. Through the detection of digital input circuit, the state is converted into a TTL signal. By measuring the signals, the time parameters can be gauged.

In order to realize batch testing for the magnetic latching relays and improve test efficiency, test device support batch access. All the main contact of relays are connected into a group in parallel, all the auxiliary contact of relays are connected into a group in parallel, each parallel contact is connected with a set of electronic combination switch, as shown in Figure 4. By selecting the switch position of the electronic combination switch, the test items are selected as contact resistance test or relay time parameters test. The number of relays can be determined according to need. The portable test device can test 12 magnetic latching relays at the same time.

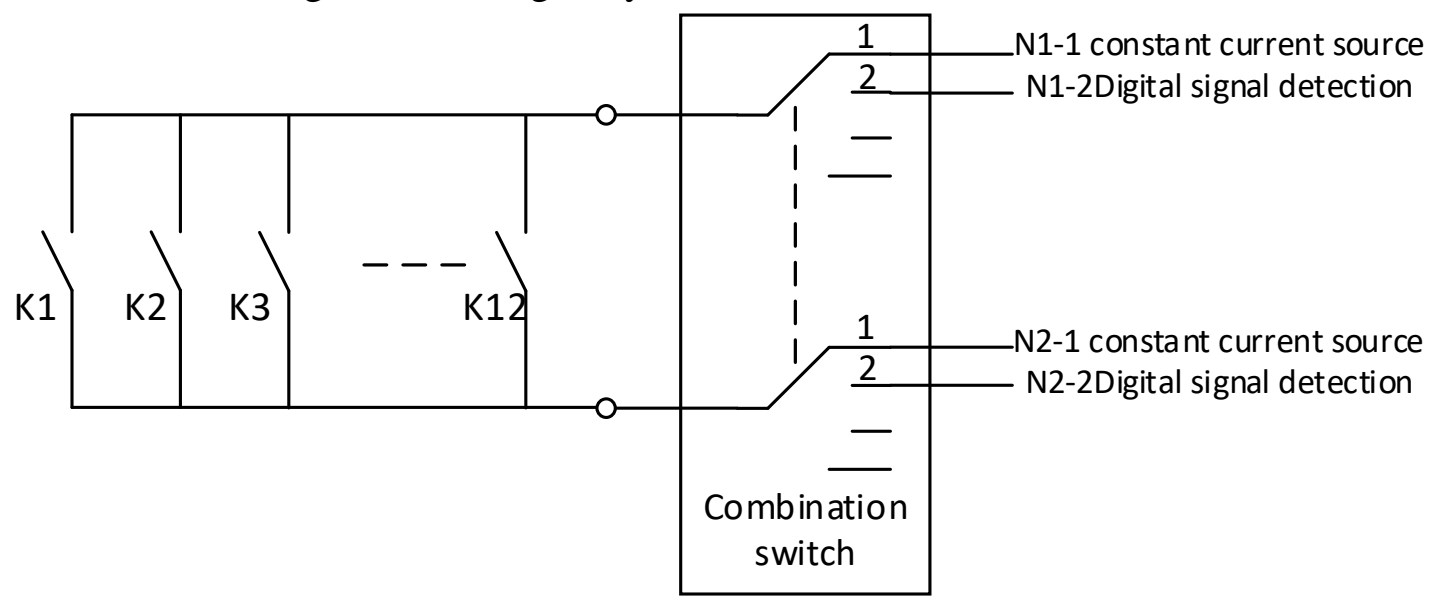

Fig.4. Schematic diagram of batch testing

Compared with the existing relay testing device, the hardware circuit of the portable test device is simple in design, high in modularization, and the design principle is mature and reliable. Therefore, portable testing device has the advantages of stable testing performance, high testing efficiency, small size, light weight, easy to carry and so on. It is especially suitable for field testing. 


\section{Software Implementation}

The software of the batch testing device for magnetic latching relays includes two parts: function software and management software.

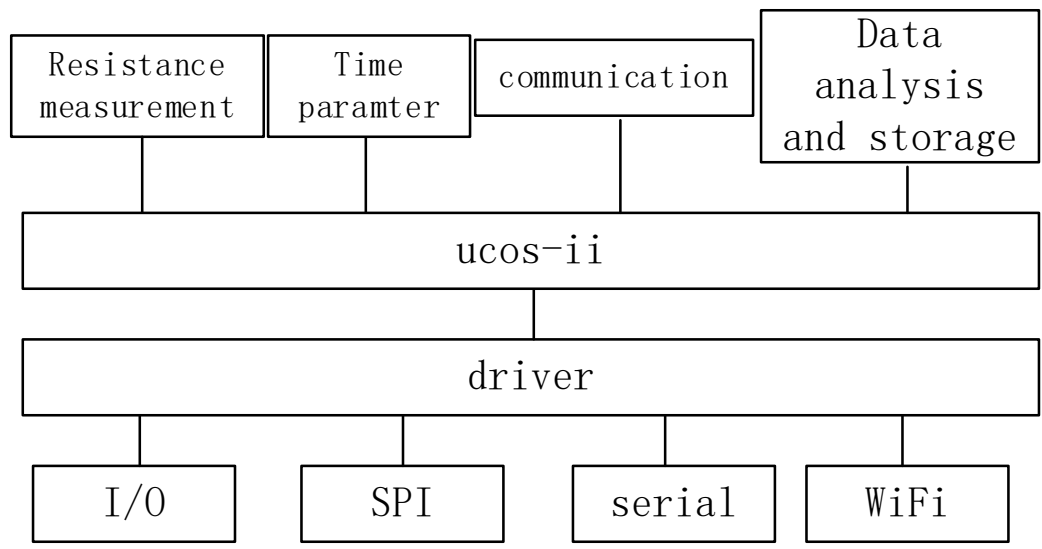

Fig.5. Functional software architecture diagram

The function software diagram is shown in Figure 5. The function software runs on the CPU chip of the device, using open source embedded operating system ucso-ii. The software uses a modular and open architecture, composed of two parts of driver and application functions. The driver software includes I/O driver, SPI driver, serial port driver and WiFi driver. The application software consists of resistance measurement module, time parameter measurement module, data analysis and storage module and communication module. The test software mainly realizes the relay contact resistance test and the time parameter test function.

The device tests the contact resistance by collecting current and voltage. First, the software sends a set instruction to the relay, then turn on the constant current source. While the current and voltage are stable, the software begin to measure the voltage of these two channels, then calculate and storage resistance. The next step is to disconnect the constant current source and sends a reset command to the relay. The other parallel relays are tested in sequence. Once completed, the average value and the tolerance value of those relays are calculated, and the result is sent to the terminal equipment.

The function software adopts the principle of capture to measure relay time parameters, including: action time, action bounce time, release time and release bounce time. The test principle as shown in Figure 6. At t0, CPU begin to capture rising edge of contact signal. At t1 , CPU have capture the first rising edge, so the $\mathrm{t} 1-\mathrm{t} 0$ is the relay action time; At last CPU captured the last rising edge at $\mathrm{t} 2$.So $\mathrm{t} 2-\mathrm{t} 1$ is the relay action bounce time. Similar, $\mathrm{t} 4-\mathrm{t} 3$ is relay release time, $\mathrm{t} 5$ - $\mathrm{t} 4$ is release bounce time.

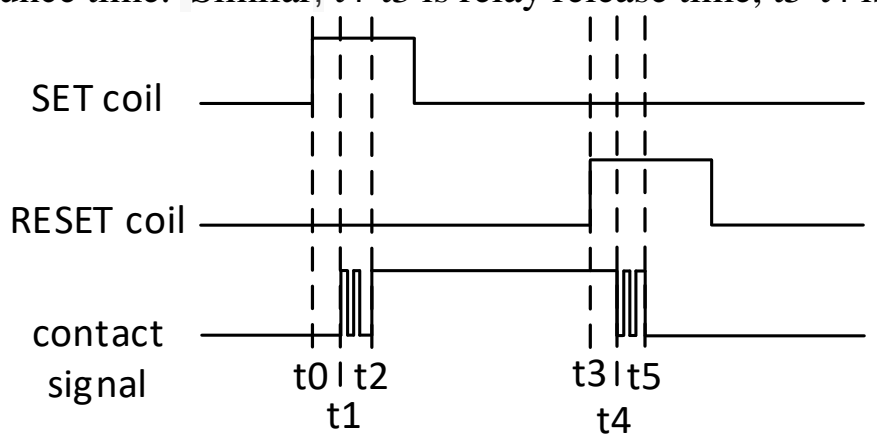

Fig.6. Sketch map of action time measurement

The management software of the device runs on the terminal equipment. It adopts the configuration mode. Different test items are implemented by different processes, and users can flexibly select test items and test flow according to the requirements. The management software has the function of database management to meet the needs of user's debugging, testing and storage of test reports. The test flow of the test device is shown in Figure 7. 


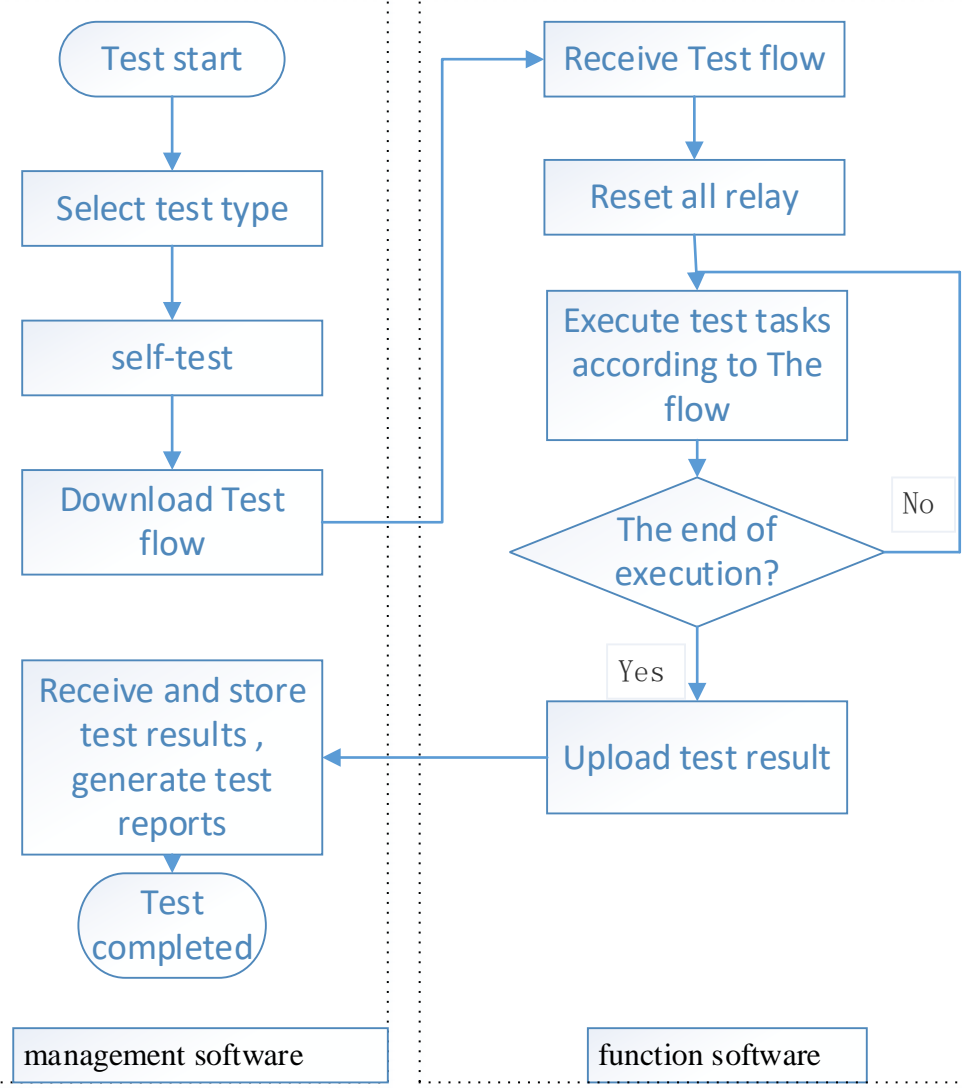

Fig.7. The flow of the software

\section{Test Result}

In order to verify the effect of portable magnetic latching relay batch testing device, two kinds of magnetic latching relays, model $\mathrm{A}$ and model $\mathrm{B}$, are selected to test time parameters and contact resistance. The parameter of Model A is 90A/250VAC, the parameter of Model B is 120A/250VAC.

Time Parameters Test. Relay Time parameters test requires the reset operation of the relay which is connection state of the original factory. After the reset, all contacts of the relays are in the state of disconnection. Execution action time testing procedures one by one, up to 12 sets of relay were tested. One set of test data records are shown in table 1.

Table 1 Test results of time parameters

\begin{tabular}{ccccc}
\hline Type & $\begin{array}{c}\text { Action time } \\
{[\mathrm{ms}]}\end{array}$ & $\begin{array}{c}\text { Action bounce time } \\
{[\mathrm{ms}]}\end{array}$ & $\begin{array}{c}\text { Release time } \\
{[\mathrm{ms}]}\end{array}$ & $\begin{array}{c}\text { Release bounce } \\
\text { time } \\
{[\mathrm{ms}]}\end{array}$ \\
\hline $\mathrm{A}$ & 6.12 & 0.34 & 3.49 & 0.00 \\
\hline $\mathrm{B}$ & 6.42 & 0.40 & 4.03 & 0.00 \\
\hline
\end{tabular}

Use oscilloscope to capture the action time, action bounce time, release time and release bounce time of the relay Type A, the wave shapes of the relay coil voltage and the contact signal are shown in Figure 8. Channel 1 is relay coil voltage signal, channel 2 is contact signal. 

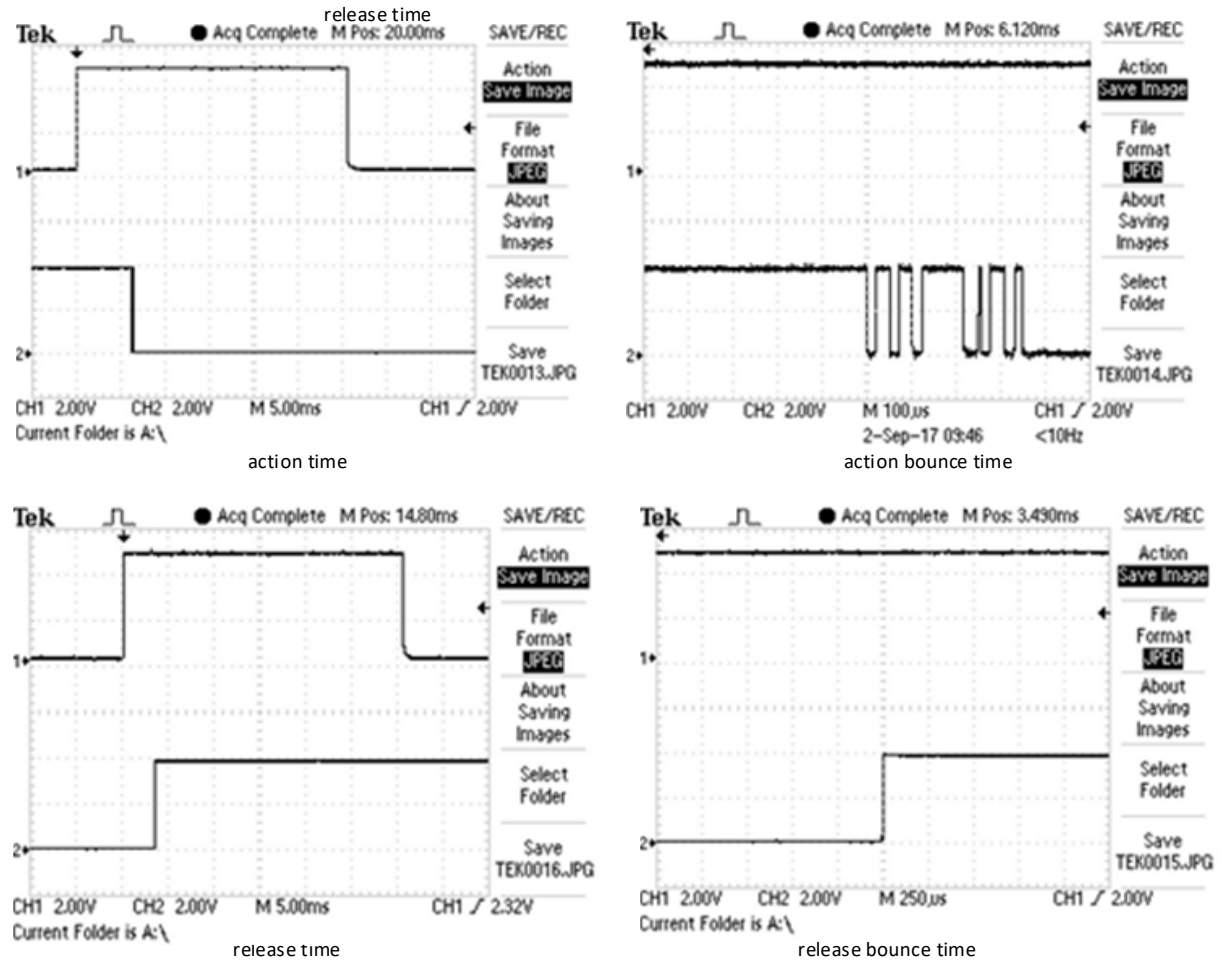

Fig.8. Coil movement time measurement

As can be seen from figure 8, the action time of type $A$ is about $6 \mathrm{~ms}$, bounce 6 times, and the length of bouncing is about 350us. The release time of type $A$ is about $4 \mathrm{~ms}$, and no rebound can be found, so the bounce time is 0s. Through comparison of Table 1 and Fig.6, we found that test results of the device are reliable, and the precision meet the requirements.

Contact Resistance Test. Using the device to execute the contact resistance test program, 12 magnetoresistive relays are tested one by one. Each relay is continuously measured and calculated 6 times, and the contact resistance of each relay is recorded. One set of test data records was selected and shown in table 2 .

Table 2 Test results of contact resistance [mohm]

\begin{tabular}{ccccccc}
\hline $\begin{array}{l}\text { Typ } \\
\mathrm{e}\end{array}$ & 1 & 2 & 3 & 4 & 5 & 6 \\
\hline $\mathrm{A}$ & 0.34 & 0.34 & 0.33 & 0.34 & 0.34 & 0.34 \\
\hline $\mathrm{B}$ & 0.33 & 0.34 & 0.32 & 0.33 & 0.33 & 0.34 \\
\hline
\end{tabular}

From the data listed in Table 2 can be seen, the contact resistance variation of Type A relay and Type B relays are very small, and the relay contact resistance of type B is smaller than type A, which is in line with the manual.

\section{Conclusion}

This paper describes the design of a portable magnetic latching relay batch testing device, introduces the system architecture, design principle of hardware circuit and the software testing process, and verify the test results of test device by building a testing system.

Practice has proved that the design of the portable magnetic latching relay testing device has wiring simple, high accuracy, high reliability, portability and can adapt to complex filed working environment. The device meets the needs of field test of magnetic latching relay users. The technology is feasible and there will be a prosperous promotion. 


\section{References}

[1] Dezhi Xiong, Xiangqun Chen, Jie Yang, Jinbo Li, Junhua Hu, Yang Xue, Reliability test method research of latching relay used in meter, [J]. Electrical Measurement \& Instrumentation. 53 (20) 122-128.

[2] Qiang Zhang, Zhongwei Yu, Hongda Liu, Automatic Testing Device for Electrical Parameters of Voltage Relay, [J]. Low Voltage Apparatus. 2010 (10) 30-34.

[3] Mingyi Fang, Xinchun Chen, Jingran Liu, Xiaohui He, Jingwei Zhang, Jingtao Feng, Study and application of relay test equipment. [J]. Modern Electronics Technique. 35 (8) 185-187.

[4] Feng Ji, Xing-xin Guo, Qing Xu, Qi-feng Huang, Research on Testing Method of Smart Meter Latching Relay, [J]. Electrical Measurement \& Instrumentation. 50 (575) 18-22.

[5] Xin Guan, Wenhua Li, Jiangfeng Shen, Linlin Zhang, Research on Testing Technology of Parameters for Railway Relay, [J]. Electrical \& Energy Management Technology. 2016 (11) 7-12. 